\title{
Combination of inspiratory and expiratory muscle training in same respiratory cycle versus different cycles in COPD patients: a randomized trial
}

Wenhui $\mathrm{Xu}^{1+}$, Rui $\mathrm{Li}^{1+}$, Lili Guan ${ }^{2+}$, Kai Wang ${ }^{1}$, Yuhe Hu${ }^{1}$, Limei Xu' ${ }^{1}$ Luqian Zhou ${ }^{2}$, Rongchang Chen ${ }^{2 *}$ and Xin Chen $^{1 *}$ (iD

\begin{abstract}
Background: Difference between combined inspiratory and expiratory muscle training in same respiratory cycle or different cycles remained unclarified. We explored the difference between both patterns of combined trainings in patients with COPD.

Methods: In this randomized, open-label, controlled trial, stable COPD subjects trained for 48 minutes daily, for 8 weeks, using a monitoring device for quality control. Ninety-two subjects were randomly and equally assigned for sham training, inspiratory muscle training(IMT), combined inspiratory and expiratory muscle training in same cycle(CTSC) or combined inspiratory and expiratory muscle training in different cycles(CTDC). Respiratory muscle strength, as the primary endpoint, was measured before and after training. Registry: ClinicalTrials.gov (identifier: NCT02326181).

Results: Respiratory muscle training improved maximal inspiratory pressure(PImax), while no significant difference was found in PImax among IMT, CTSC and CTDC. Maximal expiratory pressure(PEmax) in CTSC and CTDC was greater than $\operatorname{IMT}(P=0.026$, and $P=0.04$, respectively) and sham training $(P=0.001)$. IMT, CTSC, and CTDC shortened inhalation and prolonged exhalation $(P<0.01)$. Subjects with respiratory muscle weakness in IMT and CTDC exhibited greater increase in PImax than those without. IMT, CTSC and CTDC showed no difference in symptoms and quality of life scales among themselves $(P>0.05)$.

Conclusion: Both patterns of CTSC and CTDC improved inspiratory and expiratory muscle strength, while IMT alone only raised PImax. Respiratory muscle training might change the respiratory cycles, and be more beneficial for COPD patients with inspiratory muscle weakness.
\end{abstract}

Keywords: Chronic obstructive pulmonary disease, Inspiratory muscle training, Expiratory muscle training, Combined respiratory muscle training

\footnotetext{
* Correspondence: chenrc@vip.163.com; chen_xin1020@163.com

${ }^{+}$Wenhui Xu, Rui Li and Lili Guan contributed equally to this work.

${ }^{2}$ Department of Respiratory Medicine, The State Key Laboratory of

Respiratory Disease, National Clinical Research Center for Respiratory Disease,

Guangzhou Institute of Respiratory Health, First Affiliated Hospital of

Guangzhou Medical University, 151 Yanjiang Road, Guangzhou 510120,

China

'Department of Respiratory Medicine, Zhujiang Hosptial, Southern Medical

University, 253 Gongye Road, Guangzhou 510282, China
}

(c) The Author(s). 2018 Open Access This article is distributed under the terms of the Creative Commons Attribution 4.0 International License (http://creativecommons.org/licenses/by/4.0/), which permits unrestricted use, distribution, and reproduction in any medium, provided you give appropriate credit to the original author(s) and the source, provide a link to the Creative Commons license, and indicate if changes were made. The Creative Commons Public Domain Dedication waiver (http://creativecommons.org/publicdomain/zero/1.0/) applies to the data made available in this article, unless otherwise stated. 


\section{Introduction}

Patients with COPD generally suffer from respiratory muscle dysfunction [1]. Severe respiratory muscle dysfunction can lead to problems such as dyspnea, hypoxemia, and decreased exercise capacity. Respiratory muscle dysfunction is closely related to the mortality of patients with COPD [2, 3]. Therefore IMT has been suggested as an important solution to decreased respiratory muscle function [4]. It has shown that IMT in patients with COPD can delay deterioration of lung function via increasing inspiratory muscle strength and endurance, which relieves dyspnea, and improves quality of life $[5,6]$.

However, in addition to impaired inspiratory muscle function, expiratory muscle fatigue may also occur in COPD. Increasing intrathoracic pressure and diminishing lung volume, expiratory muscle contraction promotes effective cough [7], which has been previously thought to be associated with airway clearance [8]. It is known that expiratory muscles are usually activated at the end of expiration in COPD patients during rest, or weight-bearing breathing [9]. And this helps to maintain respiratory function [10]. Although at present there is a few of researches examining expiratory muscle trainin$\mathrm{g}(\mathrm{EMT}), \mathrm{EMT}$ alone or the combination of EMT and IMT was recommended to strengthen inspiratory and expiratory muscles [11].

Nowadays, few studies are focusing on combined inspiratory and expiratory muscle training in COPD patients, and the effect of combined training remains unclarified. Combined respiratory muscle training has been categorized into two patterns: CTSC(training both inspiratory and expiratory muscle in same respiratory cycle) and CTDC(training inspiratory and expiratory muscle separately in different respiratory cycles). Weiner P [12] allocated COPD patients to CTDC for 3 months and found it had no additional benefit compared to IMT. In contrast, Battaglia E [13] connected the target flow inspiratory muscle trainer with the expiratory muscle trainer for CTSC, and found it significantly improved respiratory muscle function in patients with COPD. Presently, it is unclear whether CTSC and CTDC provide additional rehabilitation benefits compared with IMT alone, and whether there are differences between the two patterns of combined respiratory muscle training. Thus, the study was to explore the rehabilitation effects of CTSC, CTDC, and IMT alone, using a modified threshold respiratory muscle trainer with monitoring device which allows inspiratory and expiratory muscle training in same respiratory cycle. As IMT, but not EMT, is the recommended method for respiratory muscle training at present, EMT group wasn't established in this trial.

\section{Materials and methods Study design}

The trial was carried out at Zhujiang Hospital of Southern Medical University from January 2015 to December 2017. The protocol was approved by the Zhujiang Hospital ethics committee(number: 2016-HXNK-005), and consisted with the Declaration of Helsinki. Informed consents were signed and obtained from all the subjects before the trial. Prior to the trial, it was registered on ClinicalTrials.gov (identifier: NCT02326181). Report of this trial was consistent with the Consolidated Standard of Reporting Trials statement [14].

\section{Recruitment of subjects}

During a run-in period for 2 weeks, Patients with clinically stable COPD [15], naive to pulmonary rehabilitation and willing to participate were eligible. Patients got excluded if they had cognitive disorders, organ failure, malignant tumors, or metabolic diseases.

Of the 225 COPD subjects initially enrolled, 92 subjects were recruited and randomly allocated to Sham training, IMT, CTSC, and CTDC, according to a computer-generated sequence using a simple randomization method (Fig. 1). The randomization list was concealed in sequentially numbered, sealed, opaque envelopes and prepared by an independent physician not involved in subject recruitment. Each new subject was assigned a number sequentially, then the corresponding envelope was opened to decided which group they would enter. Before allocation, subjects learned all kinds of training. Subjects continued their regular medications during the study, and had the right to withdraw at any time.

\section{Study interventions}

Subjects in all groups trained daily, with each session lasting for 48 minutes per day, 7 days a week, 8 weeks. The training was performed mainly at home, each set consisting of 3 minutes of training and 2 minutes of rest. Sham training performed 16 sets of no-load respiratory muscle training daily. IMT performed 8 sets of inspiratory muscle training and 8 sets of no-load respiratory muscle training per day. CTSC performed 16 sets of combined training in one respiratory cycle daily. CTDC performed 8 sets of inspiratory muscle training and 8 sets of expiratory muscle training separately in different cycles daily.

A modified threshold trainer with a monitoring device was used (Fig. 2-A). The modified trainer consisted of a threshold inspiratory trainer(Threshold IMT, Respironics, USA) and a threshold expiratory trainer(Threshold PEP, Respironics, USA), which were connected to a tube (Fig. 2-B) with two one-way valves(pattern ID:CN201721194926.4). Threshold trainers 


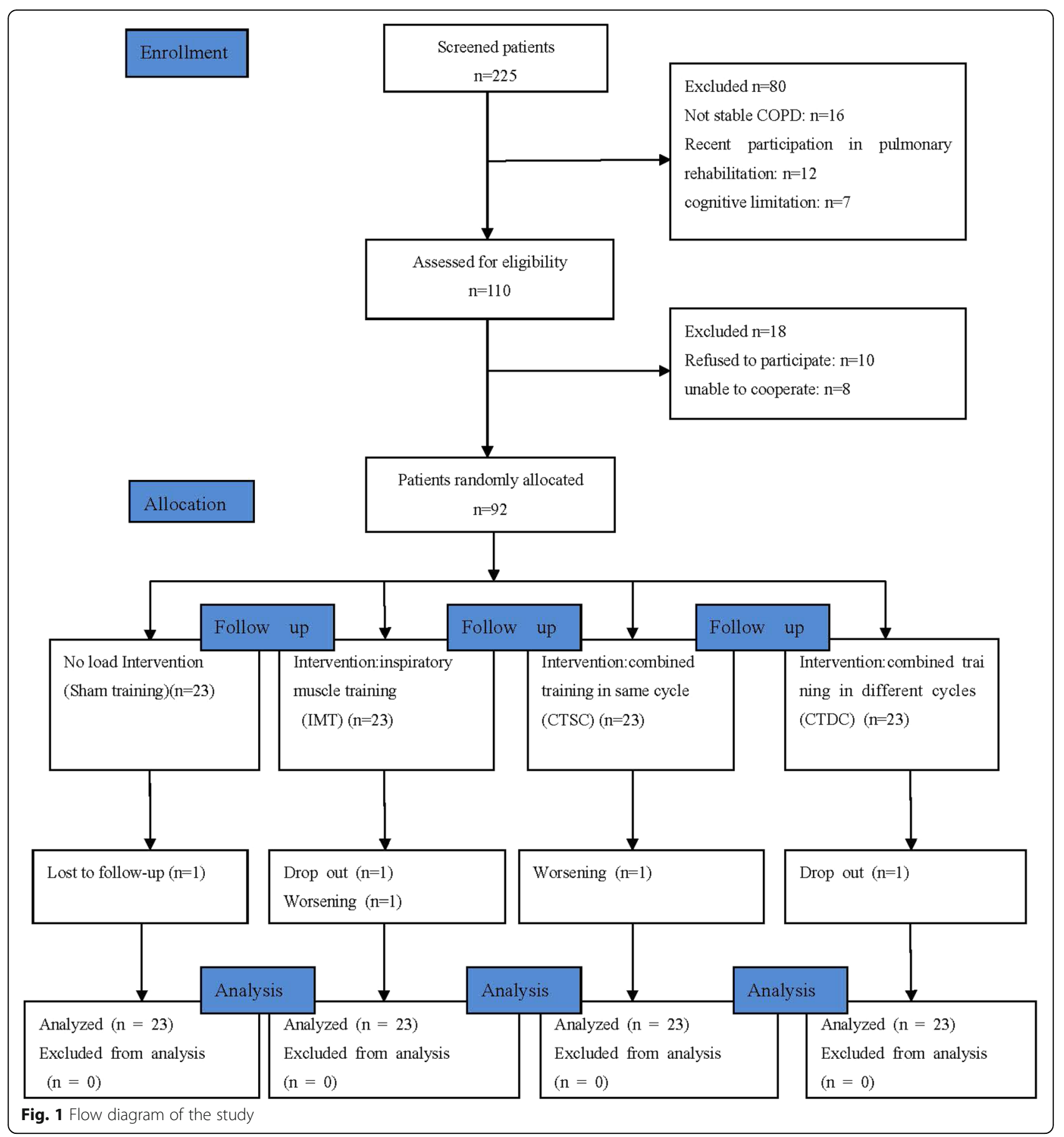

featured with an adjustable specific load [16]. Threshold PEP would be removed from the modified trainer to perform IMT. Threshold IMT would be removed to perform EMT. For sham training, both trainers would be removed. The load range of the modified trainer consisted with Threshold IMT(9-41 $\left.\mathrm{cmH}_{2} \mathrm{O}\right)$ and Threshold PEP(5-20 $\left.\mathrm{cmH}_{2} \mathrm{O}\right)$.

For training management, a monitoring device(pattern ID:CN201620070450.2) was installed (Fig. 2-C). The device recorded daily use including frequency and duration. Before training, subjects connected the device to a wireless terminal (such as a smart phone) through Bluetooth,which recorded daily use. The records were reviewed by staffs weekly. If a subject did not finish the planned assignment, they would take a lengthened training as compensation. Subjects were required to record, if any, discomfort during training. If their situation exacerbated, subjects would withdraw for treatment. Besides, 


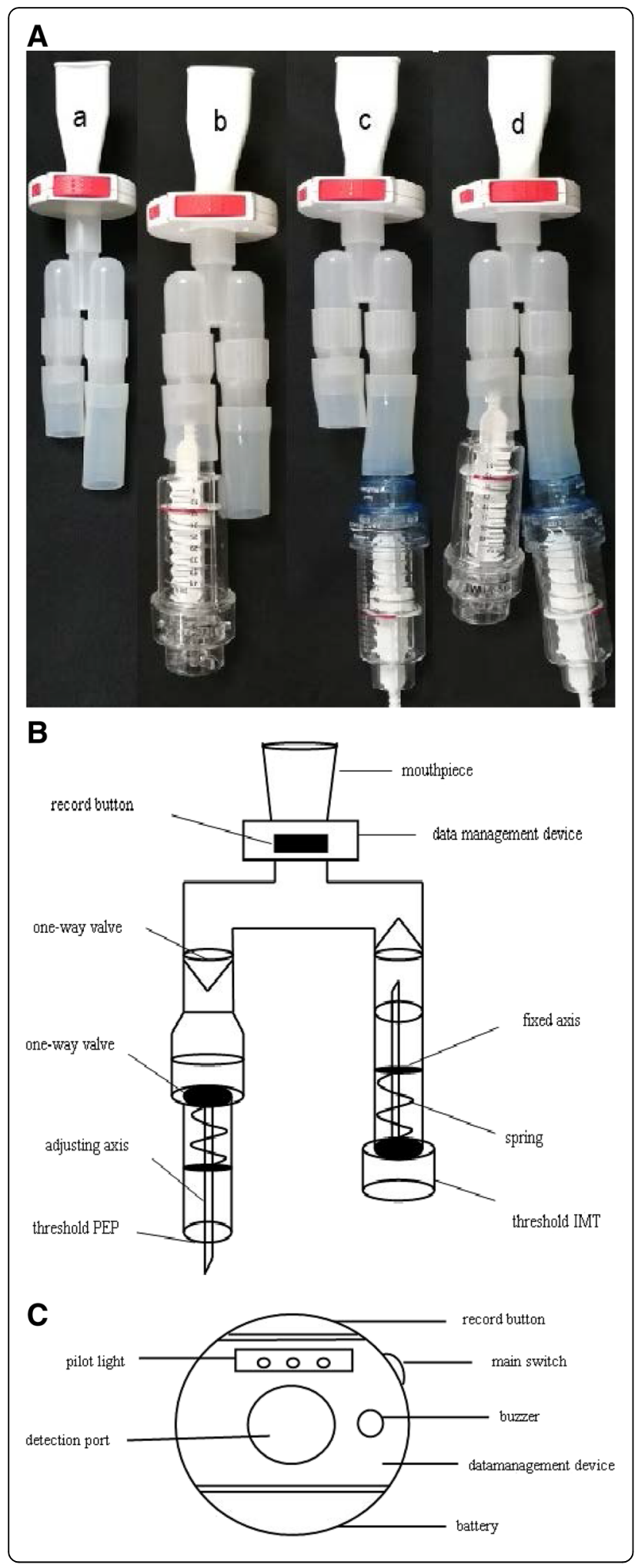

Fig. 2 A Photo of the modified trainers./a, for no-load respiratory muscle training; $b$, for inspiratory muscle training only; $c$, for expiratory muscle training only; $d$, for concurrent respiratory muscle training. B General view of the modified trainer. /Threshold PEP and Threshold IMT(Respironics, Pittsburgh, USA) were respiratory muscle trainers designed for expiratory muscle training and inspiratory muscle training, respectively. C Coronal view of the data management device. /Main switch: powering up the data management device; Record button: recording training data when pressed; Pilot light: consisting of switch indicator(turning on when main switch is pressed) and time indicator(turning on when training is completed); Buzzer: ringing when training is nearly done; Detection port: detecting the airflow passing through; Battery: for energy supply

Subjects received follow-up by telephone weekly and in the clinic every 2 weeks. In the clinic follow-up, Staffs checked the discomfort record, re-measure PImax and PEmax for load reset. The inspiratory load started at $30 \%$ PImax, and incrementally increased 5\% every two weeks until reaching 45\% PImax. The expiratory load was adjusted from 15\% PEmax plus 5\% PEmax every two weeks to $30 \%$ PEmax.

\section{Endpoints}

The primary endpoint was respiratory muscle strength. The secondary endpoints were dyspnea, breathing pattern, spirometry, exercise capacity, quality of life, emotional status, BODE index, and nutritional status. Endpoints were measured before the start of intervention (as baseline) and within 7 days after its completion. All tests were performed by one same experienced respiratory physician blinded to the allocation.

\section{Respiratory muscle strength}

Examination of respiratory muscle function consisted of measuring PImax and PEmax with a digital gauge (AZ-8205, AZ Instrument, Taiwan). Maximum value of three available tests that varied by less than $20 \%$ was recorded. The interval of each measurement lasted at 30 seconds at least. The lower limit of normal PImax was $60 \mathrm{~cm} \mathrm{H}_{2} \mathrm{O}$ [17].

\section{Dyspnea}

The modified Medical Research Council (mMRC) dyspnea scale was adopted to evaluate the severity of breathlessness.

\section{Breathing pattern}

Breath flow rate was measured using a pneumotachograph (MLT300L, ADInstruments, Australia). Respiratory rate $(\mathrm{RR})$, inspiratory time $(\mathrm{Ti})$, expiratory time $(\mathrm{Te})$, inspiratory time/total breath cycle duration (Ti/Ttot), tidal volume $(\mathrm{Vt})$, and inspiratory capacity (IC) were acquired via calculating the flow rate. 


\section{Spirometry}

Spirometry was measured using a spirometer (PonyFX 229, Cosmed, Italy) which was calibrated daily according to the 2014 ATS guidelines [18]. Forced expiratory volume in one second $\left(\mathrm{FEV}_{1}\right)$, percent-of-predicted $\mathrm{FEV}_{1}(-$ $\mathrm{FEV}_{1} \%$ ), forced vital capacity (FVC), and $\mathrm{FEV}_{1} / \mathrm{FVC}$ were recorded before a bronchodilator test.

\section{Exercise capacity}

Exercise capacity was assessed with 6-minute walk test (6MWT). During the test, heart rate and blood oxygen saturation were monitored by pulse oximetry. Oxygen saturation of all subjects was maintained at more than $90 \%$.

\section{Quality of life, emotional status}

Quality of life was reflected by St George's Respiratory Questionnaire (SGRQ) and COPD Assessment Test (CAT) [19]; Hospital Anxiety and Depression Scale (HADS) were used for evaluation of emotional status.

\section{Nutritional status}

Nutrition was reflected by BMI (body mass/height ${ }^{2}$ ) and FFMI (fat-free mass/height ${ }^{2}$ ). A FFMI $\leq 15 \mathrm{~kg} / \mathrm{m}^{2}$ in women and FFMI $\leq 16 \mathrm{~kg} / \mathrm{m}^{2}$ in men was defined as low FFMI.

\section{BODE index}

The BODE index consists of BMI (B), airflow obstruction $(\mathrm{O})$, dyspnea (D), and exercise capacity (E), which was used for a comprehensive evaluation of the subjects.

\section{Statistical analysis}

A value of $P<0.05$ was considered statistically significant. For subjects who withdrew, an intention-to-treat analysis was done. The last observation carried forward method was used for data filling. Data were presented as mean \pm standard deviation (SD) or mean \pm standard error (SE) and analyzed via SPSS 20.0 (SPSS Inc., Chicago, USA). One-way analysis of variance (ANOVA) was applied for baseline comparison. A covariance analysis (ANCOVA) was adopted to analyze the differences (after minus before, shown as $\Delta$ ) of each index, and models of least-significant difference were used for the baseline analysis. Differences in breathing patterns among groups and pre- and post-test comparisons were analyzed using mixed linear model. Subgroup analyses were performed using two-way ANOVA.

\section{Results}

Baseline characteristics

Ninety-two subjects initially participated in the study, but five dropped out. One subject in Sham training was lost to follow-up. Two subjects in IMT discontinued due to intolerance or deteriorated respiratory function, respectively. One in CTSC dropped out because of deteriorated respiratory function. One in CTDC was lost to follow-up. Basic data of all 92 subjects was not significantly different before the training $(P>0.05)$ (Table 1$)$.

\section{Respiratory muscle strength}

As shown in Table 2, the $\triangle$ PImax of IMT, CTSC, and CTDC group was significantly greater than Sham training $(P<0.05)$, but was not different among IMT, CTSC, and CTDC. Improvement of PEmax in CTSC and CTDC group was larger than IMT group $(P=0.026$, and $P=0.04$, respectively), and Sham training $(P=0.001)$, but CTSC was not significantly different from CTDC. No significant difference was found in the improvement of PEmax between IMT and Sham trainig $(P=0.218)$.

\section{Dyspnea}

The $\triangle \mathrm{mMRC}$ of IMT, CTSC, and CTDC group were significantly improved compared to Sham training $(P<$ $0.05)$. However, no significant differences among groups was shown $(P>0.05)$ (Table 2$)$.

\section{Breathing pattern}

As shown by Table 3, no significant difference was found among groups. After training, there was no significant change of breathing pattern in Sham training. CTSC, CTDC, and IMT were characterized by a decrease of $\mathrm{Ti}$ and $\mathrm{Ti} / \mathrm{Tt}$ tot, and an increase of $\mathrm{Te}$, but there were no significant differences in Vt and IC. In addition, respiratory rate in CTSC group significantly reduced.

\section{Spirometry and exercise capacity}

No significant changes were observed in $\triangle \mathrm{FVC}, \triangle \mathrm{FEV}_{1}$, $\triangle \mathrm{FEV}_{1} \%$ pred, $\Delta \mathrm{FEV}_{1} / \mathrm{FVC}$, and $\Delta 6 \mathrm{MWD}$ among groups $(P>0.05)$ (Table 2).

\section{Quality of life, emotional status}

$\triangle \mathrm{SGRQ}$ and $\triangle \mathrm{CAT}$ indicated changes in quality of life. The $\triangle$ SGRQ and $\triangle \mathrm{CAT}$ of IMT, CTSC, and CTDC were notably lower than Sham training $(P<0.05)$, but no significant difference among groups was found $(P>0.05)$. In addition, there was no significant difference in depression and anxiety scores among groups $(P=0.559)$ (Table 2).

\section{BODE index and nutritional status}

Compared with Sham training, no significant improvements was observed in BODE index, BMI, and FFMI in IMT, CTSC, and CTDC groups $(P>0.05)$ (Table 2).

Subjects with vs. without respiratory muscle weakness Each group was divided into two subgroups according to respiratory muscle strength, and then a subgroup 
Table 1 Baseline demographic and clinical characteristics

\begin{tabular}{|c|c|c|c|c|c|c|}
\hline & $\begin{array}{l}\text { Sham training } \\
(n=23)\end{array}$ & $\begin{array}{l}\mathrm{IMT} \\
(n=23)\end{array}$ & $\begin{array}{l}\text { CTSC } \\
(n=23)\end{array}$ & $\begin{array}{l}\text { CTDC } \\
(n=23)\end{array}$ & $F$ & P-value \\
\hline Age(year) & $69.43 \pm 6.44$ & $67.49 \pm 6.17$ & $68.26 \pm 7.03$ & $67.22 \pm 7.35$ & 0.498 & 0.685 \\
\hline Smoking index & $513.04 \pm 559.47$ & $356.52 \pm 389.44$ & $380.87 \pm 448.49$ & $355.22 \pm 446.89$ & 0.603 & 0.614 \\
\hline $\mathrm{BMI}\left(\mathrm{kg} / \mathrm{m}^{2}\right)$ & $20.86 \pm 4.41$ & $22.09 \pm 3.37$ & $21.86 \pm 3.03$ & $22.99 \pm 2.28$ & 1.662 & 0.188 \\
\hline FFMI $\left(\mathrm{kg} / \mathrm{m}^{2}\right)$ & $16.47 \pm 2.20$ & $15.51 \pm 2.35$ & $15.76 \pm 2.72$ & $16.82 \pm 1.38$ & 1.740 & 0.165 \\
\hline $\mathrm{PImax}>60 \mathrm{cmH}_{2} \mathrm{O}$ & $72.73 \pm 12.59(n=12)$ & $75.01 \pm 17.91(n=9)$ & $75.79 \pm 9.79(n=8)$ & $72.55 \pm 8.52(n=11)$ & 0.211 & 0.888 \\
\hline PImax $\leq 60 \mathrm{cmH}_{2} \mathrm{O}$ & $46.12 \pm 11.03(n=11)$ & $44.6 \pm 10.87(n=14)$ & $43.83 \pm 10.41(n=15)$ & $52.29 \pm 8.21(n=12)$ & 1.795 & 0.161 \\
\hline PEmax $\left(\mathrm{cmH}_{2} \mathrm{O}\right)$ & $63.82 \pm 18.46$ & $60.26 \pm 19.96$ & $56.10 \pm 16.73$ & $62.13 \pm 13.56$ & 0.844 & 0.473 \\
\hline $\mathrm{mMRC}$ & $1.609 \pm 0.839$ & $1.652 \pm 0.832$ & $1.913 \pm 0.793$ & $1.478 \pm 0.790$ & 1.155 & 0.332 \\
\hline $\mathrm{FVC}(\mathrm{L})$ & $2.312 \pm 0.781$ & $2.229 \pm 0.673$ & $2.076 \pm 0.813$ & $2.298 \pm 0.593$ & 0.517 & 0.671 \\
\hline $\mathrm{FEV}_{1}(\mathrm{~L})$ & $1.137 \pm 0.462$ & $1.161 \pm 0.325$ & $0.967 \pm 0.292$ & $1.120 \pm 0.339$ & 1.375 & 0.256 \\
\hline FEV $\%$ pred (\%) & $48.217 \pm 15.030$ & $46.652 \pm 13.506$ & $42.565 \pm 12.033$ & $47.248 \pm 12.404$ & 0.806 & 0.494 \\
\hline FEV1/FVC & $0.501 \pm 0.123$ & $0.534 \pm 0.108$ & $0.505 \pm 0.172$ & $0.495 \pm 0.112$ & 0.554 & 0.648 \\
\hline $6 \mathrm{MWD}(\mathrm{m})$ & $415.130 \pm 52.301$ & $398.957 \pm 78.733$ & $382.087 \pm 81.637$ & $400.652 \pm 78.016$ & 0.776 & 0.510 \\
\hline CAT & $10.478 \pm 5.846$ & $10.696 \pm 4.106$ & $12.609 \pm 4.131$ & $10.348 \pm 5.149$ & 1.094 & 0.356 \\
\hline SGRQ & $16.435 \pm 7.415$ & $17.348 \pm 6.184$ & $18.783 \pm 5.018$ & $17.565 \pm 7.051$ & 0.511 & 0.676 \\
\hline HADS & $5.870 \pm 3.293$ & $5.174 \pm 2.125$ & $5.696 \pm 2.835$ & $5.000 \pm 3.568$ & 0.436 & 0.727 \\
\hline BODE & $2.870 \pm 1.576$ & $3.304 \pm 1.636$ & $3.826 \pm 1.154$ & $2.783 \pm 1.930$ & 2.614 & 0.062 \\
\hline
\end{tabular}

Data are presented as mean \pm SD unless otherwise indicated

Abbreviations: IMT inspiratory muscle training, CTSC combined training in same cycle, CTDC combined training in different cycles, BMI body mass index, FFMI fatfree mass index, PImax maximal inspiratory pressure, PEmax maximal expiratory pressure, mMRC modified Medical Research Council, FVC forced vital capacity, FEV, forced expiratory volume in 1 second, \%pred percent predicted, 6MWD 6-minute walking distance, CAT COPD Assessment Test, SGRQ St George's Respiratory Questionnaire, HADS Hospital Anxiety and Depression Scale, BODE body mass index, airflow obstruction, dyspnea, and exercise capacity index

Table 2 Effects of respiratory muscle training on the IMT, CTSC, CTDC, and sham training

\begin{tabular}{|c|c|c|c|c|c|c|}
\hline & $\begin{array}{l}\text { Sham training } \\
(n=23)\end{array}$ & $\begin{array}{l}\text { IMT } \\
(n=23)\end{array}$ & $\begin{array}{l}\text { CTSC } \\
(n=23)\end{array}$ & $\begin{array}{l}\text { CTDC } \\
(n=23) \\
\end{array}$ & $F$ & $P$-value \\
\hline$\Delta \mathrm{BMI}\left(\mathrm{kg} / \mathrm{m}^{2}\right)$ & $-0.084 \pm 0.489$ & $-0.456 \pm 0.727$ & $-0.145 \pm 0.534$ & $-0.254 \pm 0.611$ & 1.540 & 0.210 \\
\hline$\Delta$ FFMI $\left(\mathrm{kg} / \mathrm{m}^{2}\right)$ & $-0.182 \pm 0.400$ & $-0.231 \pm 0.537$ & $0.128 \pm 0.913$ & $-0.070 \pm 0.664$ & 1.371 & 0.257 \\
\hline$\Delta \mathrm{PImax}\left(\mathrm{cmH}_{2} \mathrm{O}\right)$ & $2.150 \pm 4.30$ & $9.830 \pm 6.857^{\mathrm{a}}$ & $8.722 \pm 6.052^{a}$ & $8.130 \pm 6.548^{a}$ & 7.347 & $<0.001$ \\
\hline$\Delta$ PEmax $\left(\mathrm{cmH}_{2} \mathrm{O}\right)$ & $2.160 \pm 5.107$ & $4.544 \pm 4.119$ & $8.844 \pm 8.155^{a, b}$ & $8.313 \pm 6.635^{a, b}$ & 5.710 & 0.001 \\
\hline$\Delta \mathrm{mMRC}$ & $0.130 \pm 0.548$ & $-0.304 \pm 0.559^{a}$ & $-0.261 \pm 0.449^{a}$ & $-0.217 \pm 0.422^{a}$ & 3.558 & 0.018 \\
\hline$\Delta F V C(L)$ & $-0.013 \pm 0.098$ & $-0.011 \pm 0.175$ & $0.007 \pm 0.138$ & $-0.059 \pm 0.158$ & 0.706 & 0.551 \\
\hline$\Delta \mathrm{FEV}_{1}(\mathrm{~L})$ & $-0.005 \pm 0.081$ & $-0.020 \pm 0.109$ & $0.020 \pm 0.120$ & $-0.030 \pm 0.086$ & 0.561 & 0.642 \\
\hline$\Delta \mathrm{FEV}_{1} \%$ pred (\%) & $-0.261 \pm 3.427$ & $-0.826 \pm 4.549$ & $-0.174 \pm 5.789$ & $-2.030 \pm 5.358$ & 0.688 & 0.562 \\
\hline$\Delta \mathrm{FEV} 1 / \mathrm{FVC}$ & $-0.002 \pm 0.034$ & $-0.001 \pm 0.034$ & $0.014 \pm 0.049$ & $0.002 \pm 0.017$ & 0.940 & 0.425 \\
\hline$\Delta 6 \mathrm{MWD}(\mathrm{m})$ & $8.478 \pm 20.784$ & $24.174 \pm 18.364$ & $19.087 \pm 26.787$ & $19.739 \pm 12.941$ & 2.175 & 0.097 \\
\hline$\Delta \mathrm{CAT}$ & $0.304 \pm 2.098$ & $-2.304 \pm 2.787^{a}$ & $-1.435 \pm 1.903^{\mathrm{a}}$ & $-1.739 \pm 1.484^{\mathrm{a}}$ & 6.575 & 0.0005 \\
\hline$\triangle \mathrm{SGRQ}$ & $-0.174 \pm 2.103$ & $-3.087 \pm 2.429^{a}$ & $-2.565 \pm 1.927^{\mathrm{a}}$ & $-2.261 \pm 1.322^{a}$ & 9.124 & $<0.001$ \\
\hline$\Delta$ HADS & $-0.174 \pm 1.370$ & $-0.609 \pm 1.559$ & $-0.652 \pm 1.849$ & $-0.130 \pm 1.714$ & 0.692 & 0.559 \\
\hline$\triangle \mathrm{BODE}$ & $-0.174 \pm 0.778$ & $-0.478 \pm 1.163$ & $-0.348 \pm 1.434$ & $-0.174 \pm 1.230$ & 0.472 & 0.703 \\
\hline
\end{tabular}

Abbreviations: IMT inspiratory muscle training, CTSC combined training in same cycle, CTDC combined training in different cycles, BMI body mass index, FFMI fat-free mass index, PImax maximal inspiratory pressure, PEmax maximal expiratory pressure, mMRC modified Medical Research Council, FVC forced vital capacity, FEV ${ }_{1}$ forced expiratory volume in 1 second, \%pred percent predicted, 6MWD 6-minute walking distance, CAT COPD Assessment Test, SGRQ St George's Respiratory Questionnaire, HADS Hospital Anxiety and Depression Scale, BODE body mass index, airflow obstruction, dyspnea, and exercise capacity index Data are presented as mean \pm SE unless otherwise indicated; $\Delta$, difference (after minus before intervention); ${ }^{\text {a }} P<0.05 \mathrm{vs}$ control group; ${ }^{\mathrm{b}} P<0.05 \mathrm{vs}$ IMT group 
Table 3 Breathing pattern before and after respiratory muscle training

\begin{tabular}{|c|c|c|c|c|c|}
\hline & $\begin{array}{l}\text { Sham training } \\
(n=23)\end{array}$ & $\begin{array}{l}\text { IMT } \\
(n=23)\end{array}$ & $\begin{array}{l}\text { CTSC } \\
(n=23)\end{array}$ & $\begin{array}{l}\text { CTDC } \\
(n=23)\end{array}$ & $P_{2}$ \\
\hline \multicolumn{6}{|c|}{ RR(breaths/min) } \\
\hline Pre-test & 20.3 (18.6 to 21.9) & 20.3 (18.7 to 22 ) & 20.7 (19 to 22.3 ) & 20.0 (18.3 to 21.6) & \multirow{3}{*}{$P_{2}=0.949$} \\
\hline Post-test & 20.1 (18.6 to 21.6) & 20.1 (18.6 to 21.6) & 18.7 (17.1 to 20.2) & 19.8 (18.3 to 21.3) & \\
\hline$P_{1}$ & 0.584 & 0.275 & $<0.01$ & 0.466 & \\
\hline \multicolumn{6}{|l|}{$\mathrm{Ti}(\mathrm{S})$} \\
\hline Pre-test & 1.489 (1.353 to 1.624$)$ & 1.481 (1.346 to 1.617$)$ & 1.435 (1.299 to 1.570$)$ & 1.501 (1.366 to 1.637$)$ & \multirow{3}{*}{$P_{2}=0.851$} \\
\hline Post-test & $1.486(1.354$ to 1.617$)$ & 1.435 (1.303 to 1.566$)$ & $1.388(1.257$ to 1.520$)$ & 1.457 (1.325 to 1.588$)$ & \\
\hline$P_{1}$ & 0.691 & $<0.01$ & $<0.01$ & $<0.01$ & \\
\hline \multicolumn{6}{|l|}{$\mathrm{Te}(\mathrm{S})$} \\
\hline Pre-test & 1.751 (1.521 to 1.980$)$ & 1.719 (1.489 to 1.948$)$ & 1.687 (1.457 to 1.916$)$ & 1.661 (1.431 to 1.890$)$ & \multirow{3}{*}{$P_{2}=0.989$} \\
\hline Post-test & 1.755 (1.527 to 1.984 ) & 1.757 (1.528 to 1.985$)$ & $1.752(1.524$ to 1.981$)$ & 1.723 (1.495 to 1.952$)$ & \\
\hline$P_{1}$ & 0.571 & $<0.01$ & $<0.01$ & $<0.01$ & \\
\hline \multicolumn{6}{|l|}{$\mathrm{Ti} / \mathrm{Tt}$ tot } \\
\hline Pre-test & 0.466 (0.434 to 0.497$)$ & 0.474 (0.442 to 0.506$)$ & 0.476 (0.444 to 0.508$)$ & 0.488 (0.456 to 0.520$)$ & \multirow{3}{*}{$P_{2}=0.922$} \\
\hline Post-test & 0.466 (0.435 to 0.496$)$ & 0.460 (0.430 to 0.490$)$ & 0.457 (0.427 to 0.488$)$ & 0.470 (0.439 to 0.500$)$ & \\
\hline$P_{1}$ & 0.886 & $<0.01$ & $<0.01$ & $<0.01$ & \\
\hline \multicolumn{6}{|l|}{$\mathrm{Vt}(\mathrm{L})$} \\
\hline Pre-test & 0.469 (0.447 to 0.491$)$ & 0.467 (0.444 to 0.489$)$ & 0.463 (0.441 to 0.485$)$ & 0.479 (0.457 to 0.501$)$ & \multirow{3}{*}{$P_{2}=0.762$} \\
\hline Post-test & 0.465 (0.444 to 0.485$)$ & 0.471 (0.450 to 0.492 ) & 0.462 (0.441 to 0.482$)$ & 0.477 (0.457 to 0.498$)$ & \\
\hline$P_{1}$ & 0.127 & 0.127 & 0.645 & 0.539 & \\
\hline \multicolumn{6}{|l|}{$\mathrm{IC}(\mathrm{L})$} \\
\hline Pre-test & $1.613(1.461$ to 1.766$)$ & 1.568 (1.416 to 1.720$)$ & 1.541 (1.389 to 1.693$)$ & 1.665 (1.513 to 1.818$)$ & \multirow{3}{*}{$P_{2}=0.909$} \\
\hline Post-test & 1.600 (1.456 to 1.744$)$ & $1.575(1.431$ to 1.720$)$ & 1.537 (1.393 to 1.682$)$ & 1.652 (1.508 to 1.796$)$ & \\
\hline$P_{1}$ & 0.068 & 0.313 & 0.634 & 0.077 & \\
\hline
\end{tabular}

Data are presented as adjusted mean differences (95\% confidence interval of the differences) unless otherwise indicated; $P_{1}$ results of mixed linear model comparison of baseline versus week 8 in each group (within-subject effects); $P_{2}$ results of mixed linear model comparison among the four groups(between-group effects)

Abbreviations: $R R$ respiratory rate, $T i$ inspiratory time, $T e$ expiratory time, Ti/Ttot inspiratory time/ total breath cycle duration, $V t$ tidal volume, IC inspiratory capacity

analysis was performed. Respiratory muscle weakness was defined as PImax $<60 \mathrm{~cm} \mathrm{H}_{2} \mathrm{O}$. As reported in Table 4, subjects with respiratory muscle weakness in IMT group benefited more in PImax than those without $(P=0.009)$. CTDC exhibited a benefit for PImax and PEmax $(P=0.038$ and $P=0.007)$. There were not differences before and after training in all endpoints of CTSC and sham training $(P>0.05)$, except $\Delta \mathrm{FEV}_{1} \%$ pred in CTSC.

\section{Discussion}

\section{Respiratory muscle strength}

In this study, two patterns of combined respiratory muscle training, CTSC and CTDC, were compared. Both groups were not significantly different in almost all endpoints, except the significantly reduced breathing rate in CTSC. Similar to Battaglia E [13], our trial also found that combined respiratory muscle training could strengthen inspiratory and expiratory muscle. In Battaglia's work, patients received home based training twice daily for 15 minutes, 7 days a week, for 12 months with a target flow respiratory muscle trainer. The increase in PImax and PEmax was not significant until 6 months after training. However, in our study, the effects were significant after 8 weeks in CTSC and CTDC. As a target flow trainer usually requires a specific inspiratory flow which depends on breathing pattern, the outcomes may fluctuate. Nevertheless, a threshold pressure respiratory muscle trainer, independent of the flow, can regulate the training intensity precisely [16], making it more useful. Watsford M [20] applied the Powerlung device to explore the effect of CTSC on the recovery of healthy people. They adjusted the training load in accordance with subjects' endurance, and discovered that CTSC could significantly improve both PImax and PEmax. Training loads of at least 30\% PImax were required to improve respiratory muscle strength [21]. But no standard of load exists for expiratory muscle training. In our study, we used 15\% PEmax as an initial load [12] and 
Table 4 Effects of respiratory muscle training on COPD patients with or without respiratory muscle weakness

\begin{tabular}{|c|c|c|c|c|c|}
\hline Subgroups & Sham training & IMT & CTSC & CTDC & ANOVA (interaction) \\
\hline Number & 23 & 23 & 23 & 23 & \\
\hline $\mathrm{PImax}>60 \mathrm{cmH}_{2} \mathrm{O}$ & 12 & 9 & 8 & 11 & \\
\hline PImax $\leq 60 \mathrm{cmH}_{2} \mathrm{O}$ & 11 & 14 & 15 & 12 & \\
\hline \multicolumn{6}{|l|}{$\Delta \mathrm{BMI}\left(\mathrm{kg} / \mathrm{m}^{2}\right)$} \\
\hline $\mathrm{PImax}>60 \mathrm{cmH}_{2} \mathrm{O}$ & $-0.101 \pm 0.394$ & $-0.593 \pm 0.770$ & $-0.056 \pm 0.541$ & $-0.173 \pm 0.618$ & \\
\hline $\mathrm{PImax} \leq 60 \mathrm{cmH}_{2} \mathrm{O}$ & $-0.066 \pm 0.595$ & $-0.367 \pm 0.713$ & $-0.192 \pm 0.543$ & $-0.328 \pm 0.622$ & $P=0.702$ \\
\hline$p$ & 0.867 & 0.479 & 0.574 & 0.554 & \\
\hline \multicolumn{6}{|l|}{$\Delta \mathrm{FFMI}\left(\mathrm{kg} / \mathrm{m}^{2}\right)$} \\
\hline $\mathrm{PImax}>60 \mathrm{cmH}_{2} \mathrm{O}$ & $-0.188 \pm 0.431$ & $-0.232 \pm 0.583$ & $0.053 \pm 0.335$ & $0.131 \pm 0.814$ & \\
\hline PImax $\leq 60 \mathrm{cmH}_{2} \mathrm{O}$ & $-0.176 \pm 0.385$ & $-0.230 \pm 0.528$ & $0.168 \pm 1.118$ & $-0.254 \pm 0.450$ & $P=0.605$ \\
\hline$p$ & 0.941 & 0.993 & 0.780 & 0.170 & \\
\hline \multicolumn{6}{|l|}{$\Delta \mathrm{PImax}\left(\mathrm{cmH}_{2} \mathrm{O}\right)$} \\
\hline $\operatorname{PImax}>60 \mathrm{cmH}_{2} \mathrm{O}$ & $1.758 \pm 4.911$ & $5.367 \pm 5.558$ & $5.738 \pm 5.218$ & $5.218 \pm 6.244$ & \\
\hline PImax $\leq 60 \mathrm{cmH}_{2} \mathrm{O}$ & $2.576 \pm 3.710$ & $12.700 \pm 6.155$ & $10.313 \pm 6.011$ & $10.800 \pm 5.835$ & $P=0.254$ \\
\hline$p$ & 0.659 & 0.009 & 0.084 & 0.038 & \\
\hline \multicolumn{6}{|l|}{$\Delta \mathrm{PEmax}\left(\mathrm{cmH}_{2} \mathrm{O}\right)$} \\
\hline $\mathrm{PImax}>60 \mathrm{cmH}_{2} \mathrm{O}$ & $1.808 \pm 5.852$ & $4.700 \pm 3.482$ & $8.275 \pm 11.023$ & $4.600 \pm 6.340$ & \\
\hline PImax $\leq 60 \mathrm{cmH}_{2} \mathrm{O}$ & $2.544 \pm 4.404$ & $4.443 \pm 4.606$ & $9.147 \pm 6.593$ & $11.717 \pm 5.007$ & $P=0.162$ \\
\hline$p$ & 0.739 & 0.888 & 0.814 & 0.007 & \\
\hline \multicolumn{6}{|l|}{$\Delta \mathrm{mMRC}$} \\
\hline PImax $>60 \mathrm{cmH}_{2} \mathrm{O}$ & $0.167 \pm 0.577$ & $-0.333 \pm 0.707$ & $-0.250 \pm 0.463$ & $-0.273 \pm 0.467$ & \\
\hline PImax $\leq 60 \mathrm{cmH}_{2} \mathrm{O}$ & $0.091 \pm 0.539$ & $-0.286 \pm 0.469$ & $-0.267 \pm 0.458$ & $-0.167 \pm 0.389$ & $P=0.938$ \\
\hline$p$ & 0.749 & 0.847 & 0.935 & 0.559 & \\
\hline \multicolumn{6}{|l|}{$\Delta \mathrm{FVC}(\mathrm{L})$} \\
\hline PImax $>60 \mathrm{cmH}_{2} \mathrm{O}$ & $-0.033 \pm 0.114$ & $0.004 \pm 0.208$ & $0.051 \pm 0.184$ & $-0.059 \pm 0.210$ & \\
\hline PImax $\leq 60 \mathrm{cmH}_{2} \mathrm{O}$ & $0.009 \pm 0.078$ & $-0.021 \pm 0.159$ & $-0.016 \pm 0.107$ & $-0.059 \pm 0.100$ & $P=0.661$ \\
\hline$p$ & 0.312 & 0.745 & 0.277 & 0.999 & \\
\hline \multicolumn{6}{|l|}{$\Delta \mathrm{FEV}_{1}(\mathrm{~L})$} \\
\hline $\mathrm{PImax}>60 \mathrm{cmH}_{2} \mathrm{O}$ & $-0.015 \pm 0.072$ & $-0.053 \pm 0.134$ & $0.095 \pm 0.123$ & $-0.021 \pm 0.104$ & \\
\hline PImax $\leq 60 \mathrm{cmH}_{2} \mathrm{O}$ & $0.006 \pm 0.092$ & $0.001 \pm 0.089$ & $-0.021 \pm 0.101$ & $-0.039 \pm 0.068$ & $P=0.033$ \\
\hline$p$ & 0.405 & 0.749 & 0.097 & 0.687 & \\
\hline \multicolumn{6}{|l|}{$\Delta \mathrm{FEV}_{1} \%$ pred(\%) } \\
\hline $\mathrm{PImax}>60 \mathrm{cmH}_{2} \mathrm{O}$ & $-0.500 \pm 2.876$ & $-2.556 \pm 5.812$ & $2.875 \pm 4.764$ & $-1.246 \pm 6.620$ & \\
\hline PImax $\leq 60 \mathrm{cmH}_{2} \mathrm{O}$ & $0.0 \pm 4.074$ & $0.286 \pm 3.292$ & $-1.800 \pm 5.759$ & $-2.750 \pm 4.048$ & $P=0.074$ \\
\hline$p$ & 0.539 & 0.256 & 0.024 & 0.620 & \\
\hline \multicolumn{6}{|l|}{$\Delta \mathrm{FEV}_{1} / \mathrm{FVC}$} \\
\hline PImax $>60 \mathrm{cmH}_{2} \mathrm{O}$ & $-0.003 \pm 0.033$ & $-0.014 \pm 0.030$ & $0.040 \pm 0.051$ & $0.005 \pm 0.020$ & \\
\hline $\mathrm{PImax} \leq 60 \mathrm{cmH}_{2} \mathrm{O}$ & $-0.002 \pm 0.037$ & $0.007 \pm 0.035$ & $-0.001 \pm 0.044$ & $-0.001 \pm 0.014$ & $P=0.037$ \\
\hline$p$ & 0.981 & 0.148 & 0.058 & 0.451 & \\
\hline \multicolumn{6}{|l|}{$\Delta 6 \mathrm{MWD}(\mathrm{m})$} \\
\hline PImax $>60 \mathrm{cmH}_{2} \mathrm{O}$ & $3.667 \pm 25.850$ & $25.556 \pm 25.793$ & $15.750 \pm 17.958$ & $15.909 \pm 7.687$ & \\
\hline PImax $\leq 60 \mathrm{cmH}_{2} \mathrm{O}$ & $13.727 \pm 12.539$ & $23.286 \pm 12.615$ & $20.867 \pm 30.928$ & $23.250 \pm 15.910$ & $P=0.775$ \\
\hline$p$ & 0.255 & 0.780 & 0.673 & 0.180 & \\
\hline
\end{tabular}


Table 4 Effects of respiratory muscle training on COPD patients with or without respiratory muscle weakness (Continued)

\begin{tabular}{|c|c|c|c|c|c|}
\hline Subgroups & Sham training & IMT & CTSC & CTDC & ANOVA (interaction) \\
\hline \multicolumn{6}{|l|}{$\Delta$ CAT } \\
\hline $\mathrm{PImax}>60 \mathrm{cmH}_{2} \mathrm{O}$ & $0.583 \pm 2.193$ & $-2.333 \pm 2.121$ & $-1.125 \pm 2.532$ & $-1.636 \pm 1.433$ & \multirow{3}{*}{$P=0.962$} \\
\hline PImax $\leq 60 \mathrm{cmH}_{2} \mathrm{O}$ & $0 \pm 2.049$ & $-2.286 \pm 3.221$ & $-1.600 \pm 1.549$ & $-1.833 \pm 1.586$ & \\
\hline$p$ & 0.518 & 0.969 & 0.581 & 0.759 & \\
\hline \multicolumn{6}{|l|}{$\Delta \mathrm{SGRQ}$} \\
\hline $\mathrm{PImax}>60 \mathrm{cmH}_{2} \mathrm{O}$ & $0.167 \pm 2.588$ & $-3.333 \pm 1.732$ & $-2.250 \pm 2.493$ & $-2.182 \pm 1.537$ & \multirow{3}{*}{$P=0.811$} \\
\hline $\mathrm{PImax} \leq 60 \mathrm{cmH}_{2} \mathrm{O}$ & $-0.546 \pm 1.440$ & $-2.929 \pm 2.841$ & $-2.733 \pm 1.624$ & $-2.333 \pm 1.155$ & \\
\hline$p$ & 0.430 & 0.706 & 0.579 & 0.791 & \\
\hline \multicolumn{6}{|l|}{$\triangle \mathrm{HADS}$} \\
\hline $\mathrm{PImax}>60 \mathrm{cmH}_{2} \mathrm{O}$ & $-0.250 \pm 1.138$ & $-0.556 \pm 1.333$ & $-1.000 \pm 1.927$ & $-0.364 \pm 1.963$ & \multirow{3}{*}{$P=0.924$} \\
\hline PImax $\leq 60 \mathrm{cmH}_{2} \mathrm{O}$ & $-0.091 \pm 1.640$ & $-0.643 \pm 1.737$ & $-0.467 \pm 1.846$ & $0.083 \pm 1.505$ & \\
\hline$p$ & 0.788 & 0.899 & 0.523 & 0.545 & \\
\hline \multicolumn{6}{|l|}{$\Delta \mathrm{BODE}$} \\
\hline $\mathrm{PImax}>60 \mathrm{cmH}_{2} \mathrm{O}$ & $-0.167 \pm 0.937$ & $-0.556 \pm 1.014$ & $-0.125 \pm 0.991$ & $-0.364 \pm 1.286$ & \multirow{3}{*}{$P=0.803$} \\
\hline PImax $\leq 60 \mathrm{cmH}_{2} \mathrm{O}$ & $-0.182 \pm 0.603$ & $-0.429 \pm 1.284$ & $-0.467 \pm 1.642$ & $0.000 \pm 1.206$ & \\
\hline$p$ & 0.964 & 0.805 & 0.598 & 0.492 & \\
\hline
\end{tabular}

Data are presented as mean \pm SE unless otherwise indicated; $\Delta$, difference (after minus before intervention)

Abbreviations: IMT inspiratory muscle training, CTSC combined training in same cycle, CTDC combined training in different cycles, BMI body mass index, FFMI fatfree mass index, PImax maximal inspiratory pressure, PEmax maximal expiratory pressure, mMRC modified Medical Research Council, FVC forced vital capacity, FEV, forced expiratory volume in 1 second, \%pred percent predicted, 6MWD 6-minute walking distance, CAT COPD Assessment Test, SGRQ St George's Respiratory Questionnaire, HADS Hospital Anxiety and Depression Scale, BODE body mass index, airflow obstruction, dyspnea, and exercise capacity index

then the load raised incrementally, which gradually increased endurance of subjects.

The improvement of PEmax in CTSC and CTDC were significantly better than IMT in our work. Similarly, Weiner $P$ [12] found IMT alone could not enhance expiratory muscle strength. Increased expiratory load was assumed to induce a larger end-expiratory lung volume (EELV) [22]. Therefore, expiratory muscle training might worsen dynamic lung hyperinflation through increasing EELV in COPD patients. But another research stated that expiratory load was not associated with lung hyperinflation [23]. EELV, or even dynamic pulmonary hyperinflation can be reliably reflected via IC [24]. No significant change in IC occurred in each group, which meant EELV did not change significantly after training. The mechanism behind airflow limitation with expiratory muscle recruitment has been yet completely clarified. Contraction of expiratory muscle during exhalation might be a nonspecific response to increased respiratory stimulus [25]. Despite lung hyperinflation, Abdominal muscle recruitment during expiration preserves fiber length and force-generating ability of diaphragm muscle for the onset of inhalation [10]. Therefore, inspiratory muscle may get fatigue if expiratory muscle dysfunction occurs.

\section{Breathing pattern}

Through recorded breathing pattern, it's a pity that we didn't find any difference in breathing patterns between groups. But the pattern in CTSC, CTDC, and IMT was characterized by a decrease of $\mathrm{Ti}$ and $\mathrm{Ti} / \mathrm{Ttot}$, and an increase of Te. Prolonged exhalation probably relieves dyspnea by decreasing dynamic pulmonary hyperinflation [26] A reduction in Ti/Ttot might increase diaphragm blood flow [27], thus taking more oxygen to important inspiratory muscles in diaphragm. But no difference was observed in VT of each group after training, and this may result from increasing contraction velocity of inspiratory muscles to maintain VT [28]. Different from CTDC, respiratory frequency of CTSC was significantly lower after training, which may be due to differences in the physiology among the groups.

\section{Dyspnea}

Neuromechanical dissociation was a popular theory to explain the dyspnea caused by respiratory muscle dysfunction [29]. Dyspnea-associated physical activity limitation is a common complaint in COPD patients with moderate to severe airflow obstruction. Avoidance of activity to relieve dyspnea leads to a sedentary lifestyle, which ultimately causes a decline in exercise capacity. Because of inspiratory muscle fatigue and inefficiencies, COPD patients might use a high proportion of PImax to inhale, which might contribute a greater sense of dyspnea [30]. Held HE [31] suggested increase of expiratory muscle strength is beneficial to relieve dyspnea and improve quality of life. Therefore, combined respiratory muscle training helps to relieve dyspnea. In the current study, degree of dyspnea in CTSC, CTDC, and IMT 
group decreased, and respiratory muscle strength of these groups increased by different degrees. This indicated that respiratory muscle training can relieve dyspnea in COPD patients via strengthening their respiratory muscle.

\section{Quality of life}

Decreased quality of life is a predictor for mortality and rehospitalization in COPD [32]. Studies have shown that both inspiratory and expiratory muscle training can improve quality of life in patients with COPD $[8,33]$. We found that the improvement of SGRQ and CAT in CTSC, CTDC, and IMT was significantly greater than Sham training, probably owing to larger reductions in dyspnea after respiratory muscle training. Though there was no differences among CTSC, CTDC, and IMT. A study showed that the correlations among SGRQ, CAT, and $\mathrm{mMRC}$ were strong [33]. Therefore, dyspnea relieving from respiratory muscle training was beneficial to the amelioration of SGRQ and CAT.

\section{Spirometry and exercise capacity}

No significant benefits for spirometry were found from respiratory muscle training. This might be related to the incompletely reversible airway obstruction and emphysema. On the other hand, It is well known that lower limb muscle dysfunction is a leading cause of decreased exercise capacity [34]. Many researchers found IMT helpful in restoring the exercise capacity of patients with COPD, but the effect didn't reach clinical significance [5, $35,36]$. We also observed no remarkable changes in exercise capacity. It was recommended that, to enhance exercise capacity, inspiratory muscle strength should increase at least by 30\% from the baseline level [37], according to which it is rational to observe no significant improvement in exercise capacity in our work. Subjects with inspiratory muscles weakness in IMT group and CTDC group exhibited greater increases in PImax, suggesting that respiratory muscle training may be more beneficial for COPD patients with impaired inspiratory strength, similar to a prior meta-analysis [5]. But no change was noted in CTSC group. It may result from some unknown physiological mechanisms, or it just takes longer for the change to become evident.

\section{Limitation}

One major limitation of this study was that it was relatively small size. Due to the limited size, subgroup analysis could not be done in each group based on the revised $A B C D$ assessment tool in 2017 Global Initiative for COPD [1]. Despite these, to the best of our knowledge, this is the first randomized controlled trial to compare combined respiratory muscle training in same respiratory cycle and different cycles with monitoring device in COPD patients. Although the monitoring device could not record threshold load, it provided reliable quality control for the training. Monitoring device enabled us to make sure that subjects finished their training as possible, thus offering a stable rehabilitation. Although the sample size was not enough for subgroup analysis, more subjects were included in our study compared to previous researches. Besides, we also used new indicators on nutrition, quality of life and breathing pattern. Even though we could not find the better training between CTDC and CTSC, we nevertheless provided evidences about combined respiratory muscle training. The effects of combined training on patients with different inspiratory muscle strengths would assist healthcare providers in establishing individualized respiratory muscle training program.

\section{Conclusion}

In this trial, we found that two patterns of combined trainings could strengthen both inspiratory and expiratory muscle, while IMT alone did not change PEmax remarkably. We also showed that respiratory muscle training might improve the breathing pattern of patients with COPD, and the effect was more remarkable in patients with inspiratory muscle weakness. As breathing frequency in CTSC group was reduced significantly, it is needed to explore physiological mechanisms among different trainings. This study suggest that patients may benefit more from both patterns of combined trainings than IMT alone.

\section{Abbreviations \\ COPD: Chronic obstructive pulmonary disease; IMT: Inspiratory muscle training; EMT: Expiratory muscle training; CTSC: Combined inspiratory and expiratory muscle training in same cycle; CTDC: Combined inspiratory and expiratory muscle training in different cycles; PImax: Maximal inspiratory pressure; PEmax: Maximal expiratory pressure; mMRC: modified medical research council; SGRQ: St George's Respiratory Questionnaire; CAT: COPD assessment test; RR: Respiratory rate; Ti: Inspiratory time; Te: Expiratory time; Ti/Ttot: Inspiratory time/total breath cycle duration; Vt: Tidal volume; \\ IC: Inspiratory capacity; FVC: Forced vital capacity; FEV ${ }_{1}$ : Forced expiratory volume in one second; $\mathrm{FEV}_{1} \%$ : Percent-of-predicted $\mathrm{FEV}_{1} ; 6 \mathrm{MWT}$ : 6-minute walk test; HADS: Hospital Anxiety and Depression Scale; BMI: Body mass index; FFMI: Fat free mass index; EELV: End-expiratory lung volume}

\section{Acknowledgements}

Special thanks to Dr. Junfang Liu, who was in charge of the concealment and assignment as third party. The staffs are also grateful to all subjects for their participation in the research. Permissions to acknowledge were obtained from all those mentioned above.

\section{Funding}

This work was funded unconditionally by Clinical Research training program of Southern Medical University (LC2016PY032), National Key R\&D Program of China (2017YFC1310601), The Guangzhou Healthcare Collaborative Innovation Major Project (201604020012), Guangzhou Innovation and Entrepreneurship Education Project of Universities (201709T26), Special Funds for the Cultivation of Guangdong College Students' Scientific and

Technological Innovation (PDJHB0101). The sponsors have no any role in design, conduct, data interpretation of the study, and preparation, review or approval of this manuscript. 


\section{Availability of data and materials}

The datasets, as well as the patent materials, used and analyzed during the current study are available from the corresponding author on reasonable request.

\section{Authors' contributions}

$X C$ is the guarantor of this research. RC, XC were responsible for study concept and design. WX, RL, LG contributed to patient recruitment and patient follow-up. KW was responsible for outcome measurement. YH, LX finished data acquisition. LZ finished data analysis. All authors contributed to the drafting of this manuscript. All authors read and approved the final manuscript.

\section{Ethics approval and consent to participate}

Prior to its start, the study protocol was in accordance with the Declaration of Helsinki, and approved by the ethics committee of Zhujiang Hospital, Southern Medical University, Guangzhou, China. The reference number for the study is 2016-HXNK-005. Informed consents about the study procedures were signed and obtained from all the subjects before the trial.

\section{Consent for publication}

Not applicable

\section{Competing interests}

The authors declare that they have no competing interests.

\section{Publisher's Note}

Springer Nature remains neutral with regard to jurisdictional claims in published maps and institutional affiliations.

Received: 3 July 2018 Accepted: 18 October 2018

Published online: 20 November 2018

\section{References}

1. Vogelmeier CF, Criner GJ, Martinez FJ, et al. Global strategy for the diagnosis, management, and prevention of chronic obstructive lung disease 2017 Report: GOLD Executive Summary. Eur Respir J. 2017;49:1700214.

2. Wang K, Zeng GQ, Li R, et al. Cycle ergometer and inspiratory muscle training offer modest benefit compared with cycle ergometer alone: a comprehensive assessment in stable COPD patients. Int J Chron Obstruct Pulmon Dis. 2017;12:2655-68.

3. Borge $C R$, Hagen $\mathrm{KB}$, Mengshoel AM, et al. Effects of controlled breathing exercises and respiratory muscle training in people with chronic obstructive pulmonary disease: results from evaluating the quality of evidence in systematic reviews. Bmc Pulm Med. 2014;14:184.

4. American College of Chest Physicians, American Association of Cardiovascular and Pulmonary Rehabilitation. Pulmonary rehabilitation: joint ACCP/AACVPR evidence-based guidelines; ACCP/AACVPR Pulmonary Rehabilitation Guidelines Panel. Chest. 1997;112:1363-96.

5. Lotters F, van Tol B, Kwakkel G, et al. Effects of controlled inspiratory muscle training in patients with COPD: a meta-analysis. Eur Respir J. 2002;20:570-6.

6. Hill K, Cecins NM, Eastwood PR, et al. Inspiratory muscle training for patients with chronic obstructive pulmonary disease: a practical guide for clinicians. Arch Phys Med Rehabil. 2010;91:1466-70.

7. Weiner $P$, Magadle R, Beckerman M, et al. Specific expiratory muscle training in COPD. Chest. 2003;124:468-73.

8. Mota S, Guell R, Barreiro E, et al. Clinical outcomes of expiratory muscle training in severe COPD patients. Respir Med. 2007;101:516-24.

9. Ninane V, Rypens F, Yernault JC, et al. Abdominal muscle use during breathing in patients with chronic airflow obstruction. Am Rev Respir Dis. 1992;146:16-21

10. Gorini M, Misuri G, Duranti R, et al. Abdominal muscle recruitment and PEEPi during bronchoconstriction in chronic obstructive pulmonary disease. Thorax. 1997;52:355-61.

11. Neves LF, Reis MH, Plentz RD, et al. Expiratory and expiratory plus inspiratory muscle training improves respiratory muscle strength in subjects with COPD: systematic review. Respir Care. 2014;59:1381-8.

12. Weiner $P$, Magadle R, Beckerman M, et al. Comparison of specific expiratory, inspiratory, and combined muscle training programs in COPD. Chest. 2003; 124:1357-64.
13. Battaglia E, Fulgenzi A, Ferrero ME. Rationale of the combined use of inspiratory and expiratory devices in improving maximal inspiratory pressure and maximal expiratory pressure of patients with chronic obstructive pulmonary disease. Arch Phys Med Rehabil. 2009;90:913-8.

14. Jainer AK, Onalaja OA. Consolidated standard of reporting trials guidelines. Am J Psychiatry. 2003;160:191-192, 192.

15. Vestbo J, Hurd SS, Agusti AG, et al. Global strategy for the diagnosis, management, and prevention of chronic obstructive pulmonary disease: GOLD executive summary. Am J Respir Crit Care Med. 2013;187:347-65.

16. Gosselink R, De Vos J, van den Heuvel SP, et al. Impact of inspiratory muscle training in patients with COPD: what is the evidence? Eur Respir J. 2011:37:416-25.

17. Evans JA, Whitelaw WA. The assessment of maximal respiratory mouth pressures in adults. Respir Care. 2009;54:1348-59.

18. Redlich CA, Tarlo SM, Hankinson JL, et al. Official American Thoracic Society technical standards: spirometry in the occupational setting. Am J Respir Crit Care Med. 2014;189:983-93.

19. Jones PW, Harding G, Berry P, et al. Development and first validation of the COPD Assessment Test. Eur Respir J. 2009;34:648-54.

20. Watsford M, Murphy A. The effects of respiratory-muscle training on exercise in older women. J Aging Phys Act. 2008;16:245-60.

21. Larson JL, Kim MJ, Sharp JT, et al. Inspiratory muscle training with a pressure threshold breathing device in patients with chronic obstructive pulmonary disease. Am Rev Respir Dis. 1988;138:689-96.

22. Taylor BJ, Romer LM. Effect of expiratory resistive loading on inspiratory and expiratory muscle fatigue. Respir Physiol Neurobiol. 2009;166:164-74.

23. Xiao SC, Lu YR, Guo HX, et al. Effect of expiratory load on neural inspiratory drive. Chin Med J (Engl). 2012;125:3629-34.

24. Stubbing DG, Pengelly LD, Morse $\mathrm{J}$, et al. Pulmonary mechanics during exercise in subjects with chronic airflow obstruction. J Appl Physiol Respir Environ Exerc Physiol. 1980;49:511-5.

25. Weiner $\mathrm{P}, \mathrm{McC}$ (onnell $\mathrm{A}$. Respiratory muscle training in chronic obstructive pulmonary disease: inspiratory, expiratory, or both? Curr Opin Pulm Med. 2005:11:140-4.

26. Marin JM, Carrizo SJ, Gascon M, et al. Inspiratory capacity, dynamic hyperinflation, breathlessness, and exercise performance during the 6minute-walk test in chronic obstructive pulmonary disease. Am J Respir Crit Care Med. 2001;163:1395-9.

27. Buchler B, Magder S, Roussos C. Effects of contraction frequency and duty cycle on diaphragmatic blood flow. J Appl Physiol. 1985;58:265-73.

28. Villafranca C, Borzone $G$, Leiva A, et al. Effect of inspiratory muscle training with an intermediate load on inspiratory power output in COPD. Eur Respir J. 1998;11:28-33.

29. Parshall MB, Schwartzstein RM, Adams L, et al. An official American Thoracic Society statement: update on the mechanisms, assessment, and management of dyspnea. Am J Respir Crit Care Med. 2012;185:435-52.

30. Supinski GS, Clary SJ, Bark $H$, et al. Effect of inspiratory muscle fatigue on perception of effort during loaded breathing. J Appl Physiol. 1987;62:300-7.

31. Held HE, Pendergast DR. The effects of respiratory muscle training on respiratory mechanics and energy cost. Respir Physiol Neurobiol. 2014;200: 7-17.

32. Sanchez RH, Montemayor RT, Ortega RF, et al. Inspiratory muscle training in patients with COPD: effect on dyspnea, exercise performance, and quality of life. Chest. 2001;120:748-56.

33. Ringbaek T, Martinez $G$, Lange P. A comparison of the assessment of quality of life with CAT, CCQ, and SGRQ in COPD patients participating in pulmonary rehabilitation. Copd. 2012;9:12-5.

34. Debigare $R$, Maltais $F$. The major limitation to exercise performance in COPD is lower limb muscle dysfunction. J Appl Physiol. 2008;105:751-3 755-757.

35. Bavarsad MB, Shariati A, Eidani E, et al. The effect of home-based inspiratory muscle training on exercise capacity, exertional dyspnea and pulmonary function in COPD patients. Iran J Nurs Midwifery Res. 2015;20:613-8.

36. Hill K, Jenkins SC, Philippe DL, et al. High-intensity inspiratory muscle training in COPD. Eur Respir J. 2006;27:1119-28.

37. Hill K, Jenkins SC, Hillman DR, et al. Dyspnoea in COPD: can inspiratory muscle training help? Aust J Physiother. 2004;50:169-80. 